\section{Pathogenic microorganisms targeted by complex cellular communications}

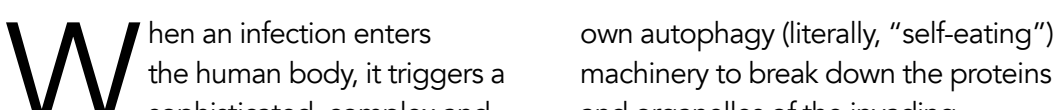 and organelles of the invading
microorganism. targeted immune response. The invaders} can take many forms - bacteria, viruses, parasites - but whatever the challenge, a response. As part of the first line of defence macrophages a name that comes from the Greek term for "big eaters") are a vital component of the immune response. In a process called phagocytosis, these large white blood cells locate, engulf and destroy threats to the body. These threats can include pathogenic bacteria and other invading microorganisms, foreign substances, cancer cells and cellular debris.

Extensive research has shown that phagocytosis involves a complex yet highly coordinated cascade of and lipids on the macrophage cell surface work together to captinto bactellum and draw macrophage, numerous organelles and proteinlipid interactions result in pathogenic bacterial clearance in a process called xenophag Xenophagy, an innate componen of the immune system, makes
use of the cell's

\section{VADING THE MACROPHAGE}

important defenders. When this happens people can develop chronic illnesses that fail to respond to treatment. For example, patients with diseases such as Chronic Obstructive Pulmonary Disease (COPD) can develop sepsis as a result of infection by drug-resistant bacteria like Streptococcus pneumoniae or Pseudomonas aeruginosa. Sepsis is a life threatening condition that can cause multiple organ failure, and is triggered by the body's own overwhelming immune response to an infection. Sepsis remains to a a a h helth concern.

To better understand how some pathogens are able to evade the body's immune defences, Dr Sen and her the immune system is maintained. Macrophages need to be primed and maintained in a constant state of readiness if they are to be effective. Key to this process is a protein gets the better of a macrophage,

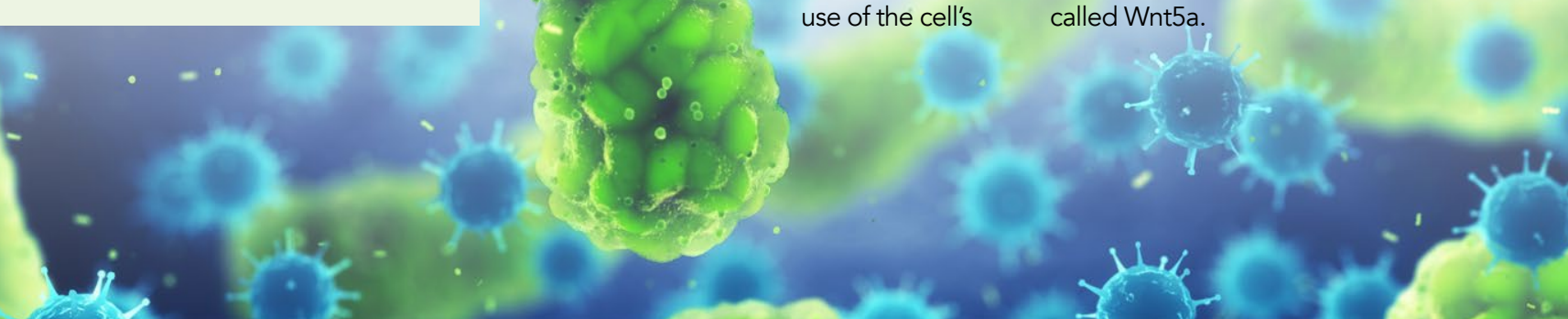

THE ROLE OF WNT5A

\section{IN THE IMMUNE RESPONSE}

secreted glycoproteins that are a family of transmitting cellular signals. Wht5a binds to two proteins that transverse the cell membrane: either the protein Frizzled (Fz) or a type of cell-surface receptor protein called ROR, or both. In the early stages of mammalian development, Wnt5a is involved in cell growth and differentiation. During this time, Wnt5a plays a vital part in cell migration and cytoskeletal reorganisation (arranging and supporting the internal structure of the cell). Dr Sen and her colleagues decided to investigate whether Whita might contribute to the process of phagocytosis in a similar way.

In 2012, Dr Sen and her team published the results of a study with that showed that signalling becteria Wnt5a and Fz plays an important part in the phagocytosis of bacteria where bacteria are "eaten" by the macrophage while being broken dow and thus rendered harmless. The team demonstrated this by creating two models: one where Wnt5a production was inhibited and one where the Wnt5a receptor $F z 5$ was blocked. In both cases, uptake of bacteria into the macrophage was inhibited. In a follow up study, published recently, Dr Sen and her team demonstrated the effect of Wnt5a-F25 signalling on phagocyto of pathogenic bacteria.

\section{THE IMPORTANCE OF SIGNALLING} It was important for $\mathrm{Dr}$ Sen and her team to investigate the mechanisms by which macrophages are primed to tackle infection. This immune homeostasis, whereby immune cells are maintained in a steady state of surveillance for to swiftly and effectively tackle infection.

Dr Sen and her colleagues established that basal (i.e. constant, low-level) Wnt5 a signalling both keeps macrophages prepared to peform their innate inmune functions and supports their survival. The team found hat the recognition and internalisation of bacteria and viruses Wnt5 party denent signaling system.

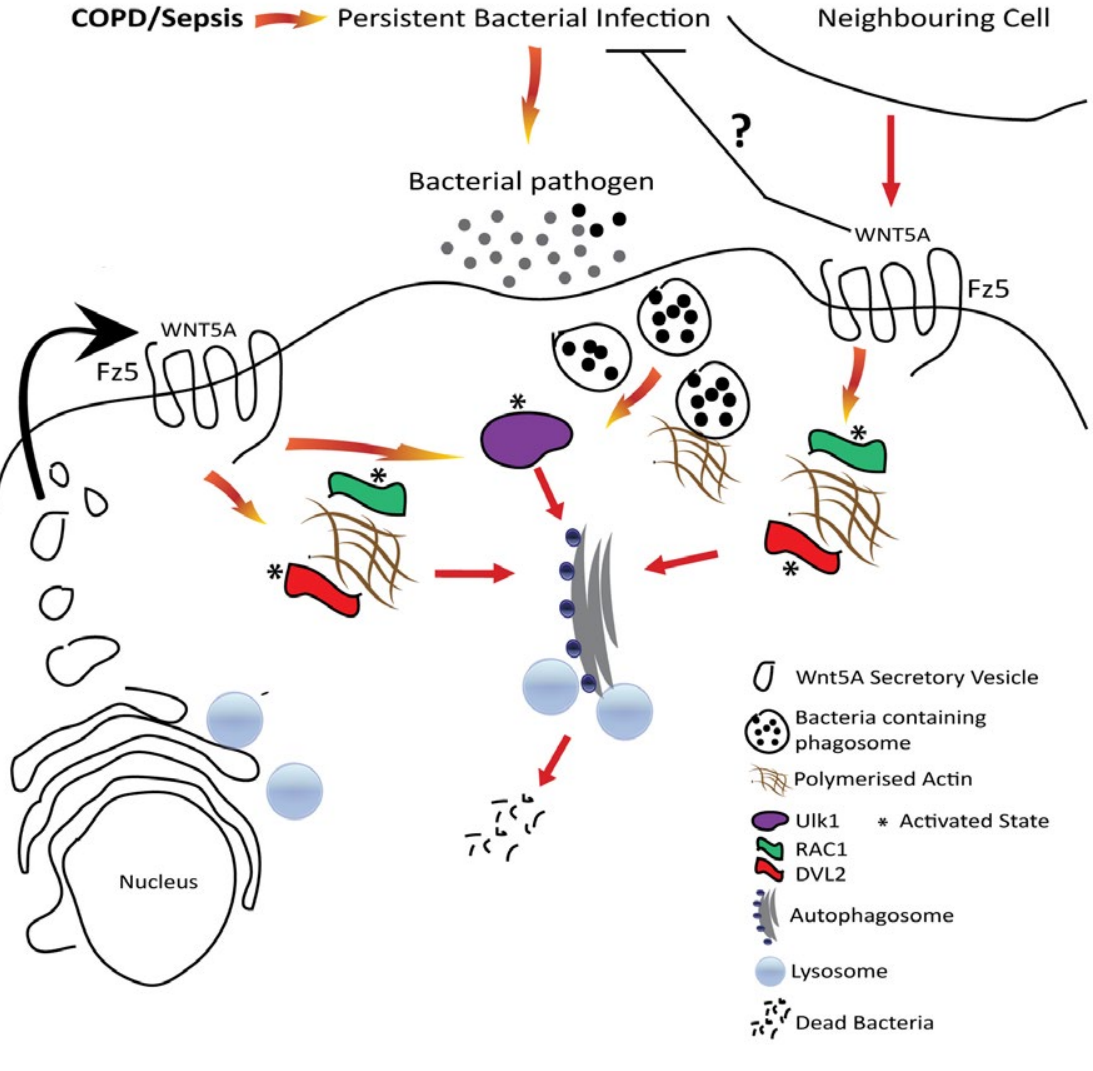

Wnt5a-F25 Signalling in macrophages promotes
interactions among its signalling intermediates.

Wnt5a plays a vital part in cell migration and cytoskeletal reorganisation.

\section{WNT5A AND LEISHMANIASIS} While establishing the crucial role of Wnt5a in tackling pathogenic bacteria the team decided to broaden the scope her colleagus. $\mathrm{mublish}$, $\mathrm{d}$ their findings on the role of 15 s signaling in ings by the parasite Leishmania donovani L donovani causes the most severe form of the disease leishmaniasis, known as visceral leishmaniasis or "black fever." This condition is the second-largest parasitic killer in the world (after malaria) and causes sy wh or

Leishmania donovani is a cunning

Wnt5a gene encodes Wnt5a protein

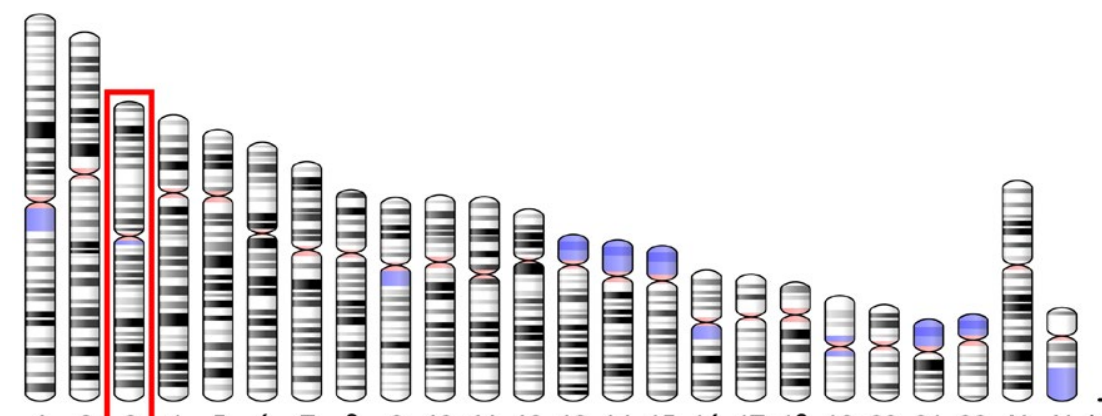

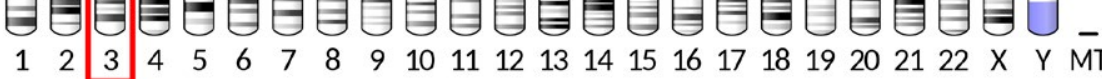


immune defences but to actually use those defences to survive. When the parasite creats a protectivani, or niche, for itself. These sofes spaces are termed parasitophorous vacuoles (PV) Sequestered in its parasite niche, L. donovani is safe from the digestive mechanisms that would normally destroy microorganisms within the macrophage. However, exactly how L. donovani and other parasitic infections form and sustain PV and avoid destruction remains unclear.

The key to the puzzle may be found in the cytoskeleton, the structure that provides mechanical support and maintains internal organisation of the cell. There is an established link between bacterial pathogenesis (the way in which bacter a dynamics of the host cell. The proten Wnt5a is also known to he protein relationship with the cytoskeleton $\mathrm{Dr}$ Sen and her team, therefore theorised that Wnt5a signalling could influence the formation of $\mathrm{PV}$ by $\mathrm{L}$. donovan

Dr Sen and her colleagues observed a decrease in Wnt5a in macrophages infected by $L$. donovani. The parasite Ilkely achieves this by suppressing Wnt5a signalling in the host cell and/or through the use of its proteases, enzymes that can break down host proteins. The consequent reduction in Wnt5a protein could cause alterations to the macrophage cytoskeleton and disrupt hron be hostasis. L. donovaniwould the be ho to take advantage of the niche within the macrophage.
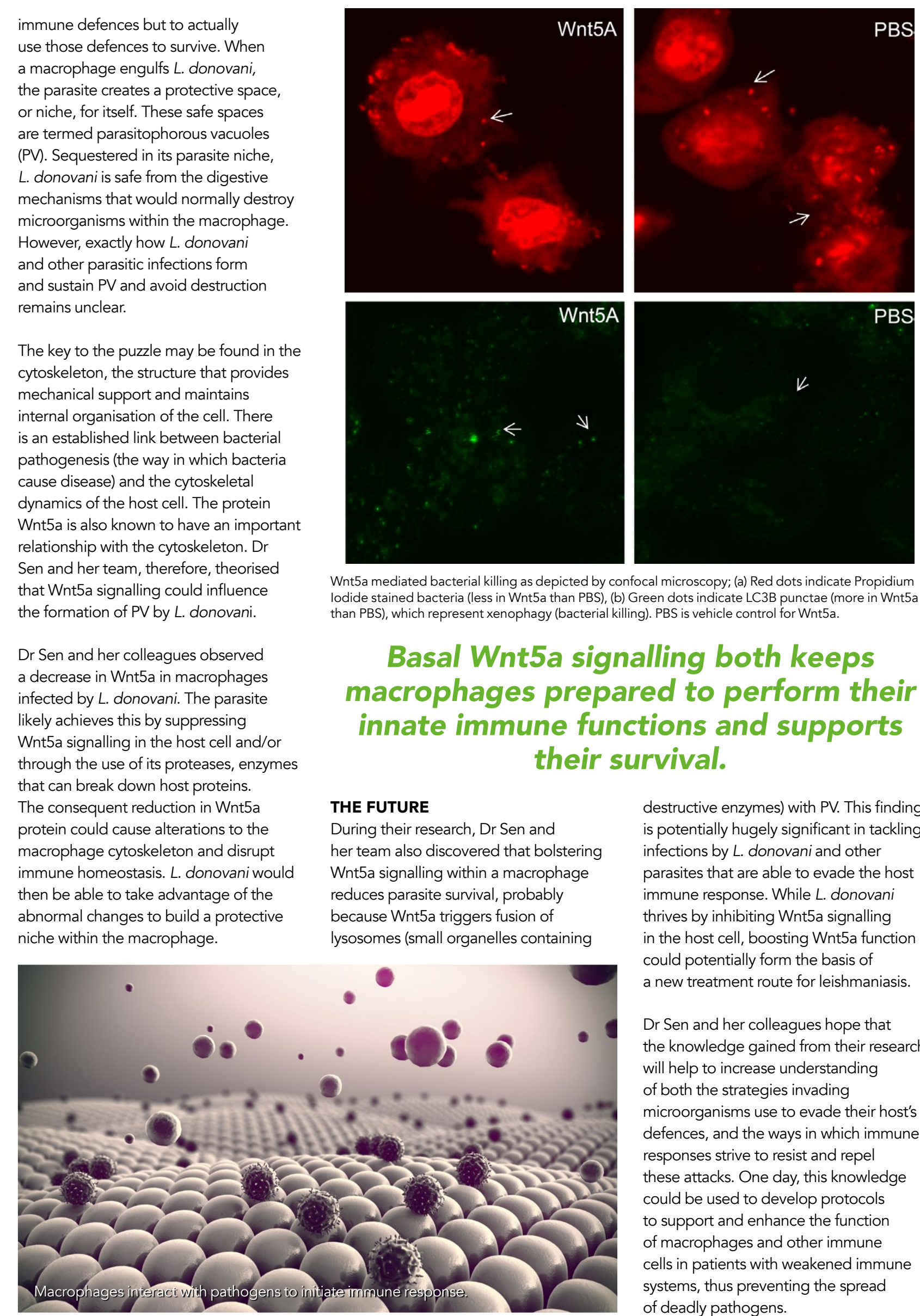

Wnt5a mediated bacterial $k$ illing as depicted by confocal microscopy; (a) Red dots indicate Propidium
lodide stained bacteria (less in Wnt5a than PBS), (b) Green dots indicate LC 38 punctae (more in Wnt5 5 a PBS), which represent xenophagy (bacterial killing). PBS is vehicle control for Wn

Basal Wnt5a signalling both keeps macrophages prepared to perform their innate immune functions and supports their survival.

\section{THE FUTURE}

ir research, Dr Sen and Wht5 signalling within a that bolstering reduces parasite sunival, probably because Wnt5a triggers fusion of

the knowledge gained from their rese will help to increase understanding of both the strategies invading defences, and the ways in which immune responses strive to resist and repel these attacks. One day, this knowledge could be used to develop protocols to support and enhance the function of macrophages and other immune cells in patients with weakened immun systems, thus preventing
of deadly pathogens.

\section{Dehind the Research} Dr Malini Sen

E: msen@iicb.res.in E: msen648@gmail.com T: $913324995730 \quad$ W: https://doi.org/10.4049/ $\begin{array}{lll}\text { jimmunol.1302817 } & \text { W: https://doi.org/10.1073/pnas.1207789109 } & \text { W: https:///doi.org/10.4049/ }\end{array}$

\section{Research Objectives}

Dr Sen's research looks at deciphering the role of mesenchymal stem cell factors such as Whts in vario aspects of immune homeostasis in

\section{Detail}

Division of Cancer Biology \& Inflammatory Disorder Indian Institute of Chemical Biology (IICB) 4 Raja SC Mullick Road Kolkata 700032

India

Bio

Dr Sen earned her Bachelor's degree from Calcutta University, India and her PhD from Albert Einstein College of Medicine, NY. She was an Arthritis Investigator at the Dept. of Medicine, UCSD and became a faculty member in the Indian Institute of Chemical Biology, a unit of CSIR, India in 2007.

Funding

Department of Biotechnology \& Council of Scientific and Industrial Research (CSIR), India.

\section{Collaborators}

Jati, Research Scholar, IICB, India borty, Research Scholar, IICB, India Dedical School, UCSD, USA - Victor Nizer, Prossor, NIPER, India

- Victor Nizet, Professor, UCSD, USA

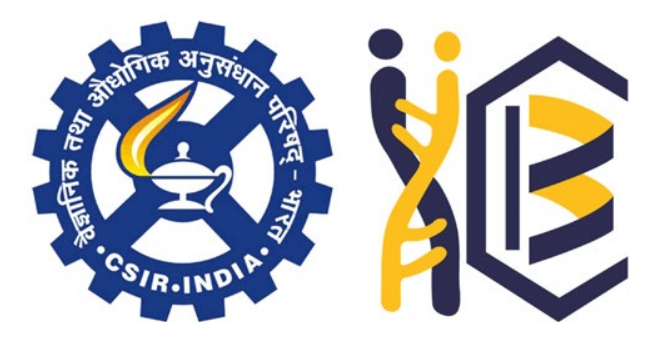

\section{References}

Chakraborty, A, Kurati, SP, Mahata, SK, Sundar, S, Roy, S and Sen, M. (2017). 'Wnt5a Signaling Promotes Host Defense against Leishmania donovani Infection'. Journal of Immunology, 199, 992-1002.

Maiti, G, Naskar, D and Sen, M. (2012). 'The Wingless homolog What atimulates phagocytosis but not bacter

Naskar, D, Maiti, G, Chakraborty, A, Roy, A, Chattopadhyay, $D$ and Sen, M. (2014). 'Wnt5a-Rac1-NF-kB Homeostatic Circuitry Sustains Innate Immune Functions in Macrophages' Journal of Immunology, 192, 4386-4397.

Jati S, Kundu S, Chakraborty A, Mahata SK, Nizet V and Sen M (2018). 'Wnt5a Signaling Promotes Defense Against Bacteria Pathogens by Activating a Host Autophagy Circuit'. Frontiers in Immunology, 9: 679. doi: 10.3389/fimmu.2018.00679.

\section{Personal Response}

What are the next steps in investigating the role of cytoskeletal dynamics in the immune response? III We are trying to dig deep into the molecular details of how Wnt5a signalling intermediates in host macrophages are intertwined with the cytoskeletal dynamics during the regard, we are focusing on the influence of Wnt5 signalling on cytoskeletal actin binding and nucleating proteins, which come into play when a functional cytoskeleton reacts to pathogen assaults. Through different experimental procedures, we hope to figure out how Wnt5a signalling
utilises the cytoskeletal dynamics to distinguish pathogen from non-pathogens during the arbitration of the host autophagy circuit for pathogen clearance, thereby resisting infection.

What are your future plans for research in this area?

W We would like to conduct experiments aimed at a detailed evaluation of the significance of Wnt signalling in the context of sepsis, which is associated with an impaired
immune system. 